\title{
Immunological profiling of key inflammatory drivers of nasal polyp formation and growth in chronic rhinosinusitis*
}

\author{
Timothy C. Biggs 1,2,3, Stephen M. Hayes, 1,2,3,4, Philip G. Harries³, \\ Raymond N. Allan ${ }^{4,5}$, Andrew F. Walls', Sylvia L.F. Pender1, Rami J. Salib1,2,3,4 \\ ' Academic Unit of Clinical and Experimental Sciences, Faculty of Medicine, University of Southampton, Southampton, United Kingdom \\ ${ }^{2}$ Southampton NIHR Respiratory Biomedical Research Centre, University of Southampton and University Hospital Southampton NHS Foundation Trust, \\ Southampton, United Kingdom \\ ${ }_{3}^{3}$ University Hospital Southampton NHS Foundation Trust, Southampton, United Kingdom \\ ${ }^{4}$ NIHR Wellcome Trust Clinical Research Facility, University Hospital Southampton NHS Foundation Trust, Southampton, United Kingdom \\ Department of Biological Sciences, Faculty of Environmental \& Life Sciences, University of Southampton, Southampton, United Kingdom
}

Rhinology 57: 0, 000 - 000, 2019

https://doi.org/10.4193/Rhin19.167

*Received for publication:

April 19, 2019

Accepted: May 17, 2019

\begin{abstract}
Background: Chronic rhinosinusitis (CRS) is a chronic inflammatory condition of the upper airways, often associated with the formation of nasal polyps (CRSwNP). It is well established that macroscopically normal (non-polypoidal) sinonasal mucosa in CRSwNP patients can undergo polypoidal change over time, turning into frank polyps. However, little is known about what drives this process. This study aimed to investigate potential drivers of nasal polyp formation or growth through comparison of the immunological profiles of nasal polyps with contiguous non-polypoidal sinonasal mucosa, from the same patients.
\end{abstract}

Methods: The immune profiles of three types of tissue were compared; nasal polyps and adjacent non-polypoidal sinonasal mucosa from 10 CRSwNP patients, and sinonasal mucosa from 10 control patients undergoing trans-sphenoidal pituitary surgery. Nasal polyp and control samples were also stimulated with Staphylococcus aureus enterotoxin B (SEB) using a nasal explant model, prior to cytokine analysis. Real time quantitative polymerase chain reaction (IL-5, T-bet, IL-17A, FoxP3, TLR-4, IL-8, IL-1 3 and IL-6) and Luminex (IFNY, IL-5 and IL-17A) were used to quantify pro-inflammatory responses.

Results: Nasal polyps and contiguous non-polypoidal sinonasal mucosa from CRSwNP patients displayed a very similar pro-inflammatory profile. When stimulated with SEB, nasal polyps displayed a Th2/Th17 mediated response when compared to controls.

Conclusions: In CRSwNP, nasal polyps and non-polypoidal sinonasal mucosa from the same patient displayed a similar pro-inflammatory profile skewed towards the Th2/Th17 pathway in nasal polyps following SEB stimulation, with evidence of disordered bacterial clearance. These factors may contribute to enhanced survival of bacteria and development of a chronic inflammatory milieu, potentially driving new polyp formation and recurrence following surgical removal.

Key words: chronic rhinosinusitis, chronic rhinosinusitis with nasal polyps, nasal polyps, Staphylococcus aureus, Staphylococcus aureus enterotoxin B

\section{Introduction}

Chronic rhinosinusitis (CRS) is an inflammatory condition of the upper airways including the nose and paranasal sinuses affecting up to $15 \%$ of the population ${ }^{(1)}$. It causes chronic symptoms including nasal obstruction, facial pain, nasal dischar- ge, and reduction in the sense of smell, significantly impacting quality of life and healthcare resources. It can be broadly classified into two main categories based on the presence (chronic rhinosinusitis with polyps, CRSwNP) or absence (chronic rhinosinusitis without polyps, CRSsNP) of nasal polyps. Nasal polyps 
affect up to $4 \%$ of the population, and up to $20 \%$ of CRS patients will develop nasal polyps at some stage in their lifetime ${ }^{(1)}$. Associated risk factors for recurrent and recalcitrant disease include asthma, allergy and aspirin sensitivity ${ }^{(1)}$. Proposed pathophysiological mechanisms in CRS include the role of superantigens ${ }^{(2-4)}$, abnormal cell-mediated immune responses ${ }^{(5,6)}$, remodelling ${ }^{(7-9)}$, epithelial defects ${ }^{(10,11)}$, osteitis of the sinus walls ${ }^{(12,13)}$, viral ${ }^{(14)}$, bacterial ${ }^{(15-18)}$, and fungal factors ${ }^{(19,20)}$. However, whilst many theories of aetiopathogenesis exist, the cause remains unclear. Historically, CRSwNP has been regarded primarily as a T-helper (Th) 2 mediated disease, characterised by IL-5 driven eosinophilic inflammation. Recently there has been a move away from the rigid Th2 classification of CRSwNP towards inflammatory endotyping, including recent evidence of a Th17 mediated response in some patients ${ }^{(21-24)}$. This raises the possibility of a spectrum of disease spanning from CRSsNP to CRSWNP(25). This may help explain why some CRS patients with seemingly macroscopically normal (non-polypoidal) sinonasal mucosa at the time of presentation can go on to develop nasal polyps in the future. It is well established that bacteria, including toxins such as Staphylococcus aureus enterotoxin B (SEB), play a role in driving a pro-inflammatory response in CRS, with the release of cytokines skewed towards a Th2 profile ${ }^{(1,3,26)}$. Recently, a novel finding of intracellular Staphylococcus aureus (S. aureus) within mast cells in nasal polyps was made ${ }^{(18)}$. Intracellular localisation of bacteria could represent a means of them evading the immune system as well as acting as a reservoir to constantly seed bacteria into the extracellular environment, thus sustaining a chronic inflammatory reaction. Furthermore, the presence of subepithelial and intracellular bacteria raises the probability of a disordered immune response against bacteria, favouring their survival with resultant pro-inflammatory effects. Evidence of such a disordered immune response is currently lacking.

Toll-like receptors (TLR) play an important role in the immune response against bacteria, recognising pathogen-associated molecular patterns (PAMPs) on the surface of microbes and activating an innate immune response against them. Furthermore, IL-8, IL- 6 and IL- $1 \beta$ propagate an immune mounted response against bacteria. IL-8, a chemotactic factor for neutrophils and mast cells drawing them to the site of infection ${ }^{(27,28)}$, and its levels have been shown to be increased in CRS ${ }^{(21,24)}$. IL-6 and IL$1 \beta$ are important in the immune mediated clearance of bacteria, and their deficiency has been associated with worsening bacterial infections in other conditions ${ }^{(29-31)}$.

This study aims to investigate potential drivers of nasal polyp formation and growth through comparison of immunological profiles of nasal polyps with contiguous areas of non-polypoidal sinonasal mucosa from the same patient. This involved the study of cytokines and transcriptional factors associated with the 4 major T-cell pathways: Th1 (T-bet and IFNY), Th2 (IL-5), Th17 (IL-17A), and Treg (FoxP3). In addition, bacterial-related toll like receptors (TLR4) and bacterial-associated cytokines (IL-8, IL-6 and IL-1 $\beta$ ) were studied, looking for evidence of a disordered immune response against bacteria.

\section{Materials and methods}

\section{Subjects}

All methods were carried out in accordance with relevant guidelines and regulations. All experimental protocols were approved by the Southampton and South West Hampshire Research Ethics Committee (ref - 09/H0501/74). Informed consent was obtained from all participants.

A total of 20 subjects ( 10 patients with CRSwNP and 10 control subjects), meeting the diagnostic criteria defined in the latest European position paper on rhinosinusitis and nasal polyps $2012^{(1)}$, were included in the study. For the cytokine gene expression study, 10 CRSwNP and 10 control patients were studied. For the nasal explant model SEB stimulation study, 10 CRSwNP patients and 5 control patients were studied.

Patients underwent functional endoscopic sinus surgery by the senior authors (PGH and RJS) at the University Hospital Southampton NHS Foundation Trust (UHSNFT), after having failed an 8-week trial of maximal medical therapy including Doxycycline, topical and/or oral steroids, and nasal saline douches. No patients had used either topical or systemic corticosteroid, antibacterial, or antihistamine therapies in the 8 weeks prior to surgery. Exclusion criteria included age less than 18 years, and patients suffering from cystic fibrosis or primary ciliary dyskinesia. All subjects were skin-prick allergy tested to a standard battery of 10 common aero-allergens (ALK-Abelló, Reading, Berkshire, United Kingdom) prior to surgery. Radiological severity of sinus disease was graded on a computed tomography sinus scan using the Lund-Mackay scoring system ${ }^{(32)}$. Preoperative demographic data were collected (Tables 1 and 2) including age, sex, smoking habit, history of asthma, atopic status, previous sinonasal surgery, and medical history. Control tissue was obtained from 10 patients with no clinical history or radiological evidence of CRS, undergoing endoscopic trans-sphenoidal pituitary surgery at the Wessex Neurological Centre, UHSNFT.

\section{Tissue specimens}

In total three types of tissue were collected and compared in the first part of the study. Nasal polyps and contiguous non-polypoidal sinonasal mucosa (middle meatus) were collected from CRSwNP patients, and sinonasal mucosal samples were obtained from the controls. Tissue specimens were placed in Hanks' Balanced Salt Solution (Gibco, Paisley, United Kingdom) and transported on ice for processing. Tissue was cut into sections $\left(3 \mathrm{~mm}^{3}\right)$, snap frozen in liquid nitrogen and stored at $-80^{\circ} \mathrm{C}$ for real time quantitative polymerase chain reaction (RT-qPCR). Fresh tissue was placed within a nasal explant model, outlined below, and processed for RT-qPCR and Luminex for the analysis of SEB. 
Table 1. Patient demographics - Cytokine gene expression study (Figure 1).

\begin{tabular}{|lcc|}
\hline \multicolumn{1}{|c}{ Characteristics } & CRSwNP & Control \\
\hline Subject numbers & 10 & 10 \\
\hline Mean age (range) & $43(31-67)$ & $56(46-75)$ \\
\hline Sex & 6 Male/4 Female & 5 Male/5 Female \\
\hline Current smokers & 3 & 0 \\
\hline Asthma & 6 & 0 \\
\hline Positive SPT & 3 & 0 \\
\hline Previous Surgery & 2 & 0 \\
\hline Mean SNOT-22 score & $62(20-101)$ & $22(5-61)$ \\
(range) & & 0 \\
\hline Mean LMS (range) & $14.5(3-24)$ & \\
\hline
\end{tabular}

SPT - Skin prick test, SNOT - Sinonasal outcome test, LMS - Lund Mackay score.

\section{Nasal explant culture model}

Polyp and control sinonasal mucosal samples were cut into small explants $\left(3 \mathrm{~mm}^{3}\right)$, with 4-pieces placed epithelial side up on a $100 \mu \mathrm{m}$ cell strainer (VWR International, Radnor, PA, USA) within a 6-well tissue culture plate (Greiner Bio-One International, Kremsmunster, Austria). Wells contained $1 \mathrm{ml}$ of serum-free synthetic interstitial fluid AQIX RS-1 medium (AQIX Ltd, London, UK). Tissue explants were semi-submerged, thereby maintaining the physiological air/fluid interface. Explants were cultured $\left(37^{\circ} \mathrm{C}\right.$ with $5 \% \mathrm{CO}_{2}$ ) for 24 hours, with and without the addition of SEB (10 $\mathrm{gg} / \mathrm{ml}$, Sigma-Aldrich Ltd, Gillingham, UK) placed within the culture medium. Tissue and culture supernatants were collected following culture, snap frozen in liquid nitrogen, and stored at $-80^{\circ} \mathrm{C}$ for future processing.

Real time quantitative polymerase chain reaction

Frozen tissue was disrupted using $1.0 \mathrm{~mm}$ zirconia beads (Thistle Scientific, Glasgow, Scotland, UK), for 45 seconds in a MagNA lyser (Roche, Basel, Switzerland) set at 4,500 rpm, and chilled on ice at $4^{\circ} \mathrm{C}$ for 5 minutes. Total RNA was isolated with an RNeasy Mini Kit (Qiagen, Hilden, Germany) as per the manufacturer's instructions. Reverse transcription was undertaken using the Thermofisher Scientific high capacity RNA-to-cDNA kit (Life Technologies Ltd, Carlsbad, CA, USA) using 500 ng of RNA product per reaction and quantified using the NanoDrop ${ }^{\circledR}$ ND-1000 spectrophotometer (Life Technologies Ltd, Carlsbad, CA, USA). Amplification reactions were performed on an $A B I 7900 H T P C R$ system (Applied Biosystems, Foster City, CA, USA), using SYBR Green PCR Master Mix (Primerdesign Ltd, Southampton, Hampshire, UK) and specific primers (Primerdesign Ltd, Southampton, Hampshire, UK; Appendix I). The amplification protocol consisted of 2 mins at $95^{\circ} \mathrm{C}, 40$ cycles of $95^{\circ} \mathrm{C}$ for 15 seconds, and $60^{\circ} \mathrm{C}$
Table 2. Patient demographics - Nasal explant model SEB stimulation study (Figures 2 and 3).

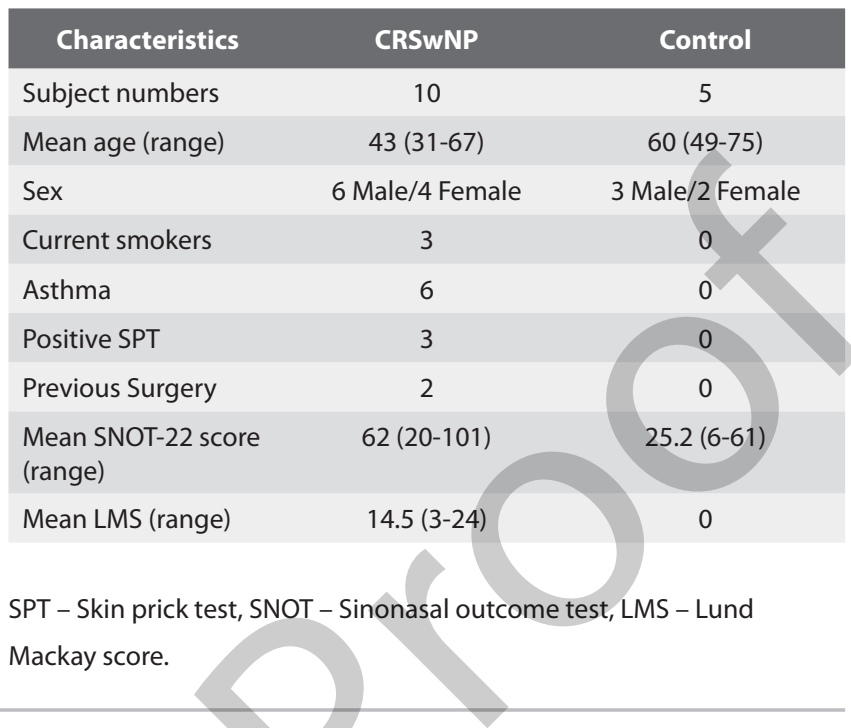

for $1 \mathrm{~min}$, followed by a melt curve analysis $\left(95^{\circ} \mathrm{C}\right.$ for 15 seconds, $60^{\circ} \mathrm{C}$ for 15 seconds, and $95^{\circ} \mathrm{C}$ for 15 seconds). The expression of a housekeeping gene, beta-actin ( $\beta$-actin), was used to normalise for transcription and amplifications variations among samples. The comparative CT method $\left(2-^{\Delta \Delta C} \mathrm{~T}\right)$ was used to calculate normalised relative fold change compared to pooled controls ${ }^{(33)}$.

\section{Measurement of supernatant cytokines (Luminex ${ }^{\circledR}$ )}

Concentrations of IFNy, IL-5, and IL-17A were measured on tissue culture supernatants using Luminex ${ }^{\circledR}$ (R\&D systems Inc, Minneapolis, MN, USA), as per the manufacturer's instructions. The plate was analysed on the Luminex analyser (Bio-Plex ${ }^{\circledR} 200$ System, Bio-Rad Laboratories Ltd, Hercules, CA, USA).

Luminex results were standardised by measuring the total protein content of samples, thereby representing $\mathrm{pg} / \mathrm{ml}$ per $\mu \mathrm{g}$ of total protein for each analyte measured. Total protein was measured using the Bio-Rad protein assay kit (Bio-Rad Laboratories Ltd, Hercules, CA, USA) as per the manufacturer's instructions. All standards and samples were undertaken in triplicates.

\section{Statistical analysis}

Statistical analysis was performed using Graph Pad Prism 6.0 software (Graph Pad Software Inc., San Diego, CA, USA). For the ex vivo tissue samples, statistical analysis was performed using a Wilcoxon test for paired samples (nasal polyp and non-polypoidal sinonasal mucosa) and Mann-Whitney U-test for all other analyses. Statistical analysis for explant model culture samples was undertaken using a paired t-test when examining cytokine gene expression ratios between SEB stimulated and unstimulated samples. Unpaired t-tests were used to examine cytokine gene expression ratios between control and nasal polyp samples, both stimulated and unstimulated. 
A.

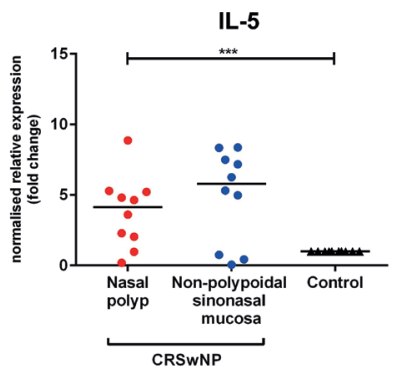

E.

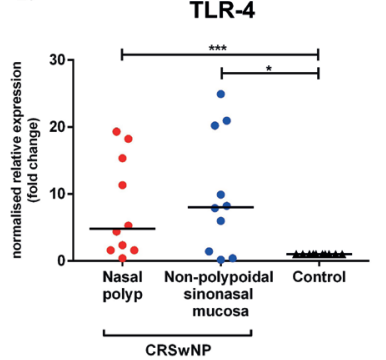

B.

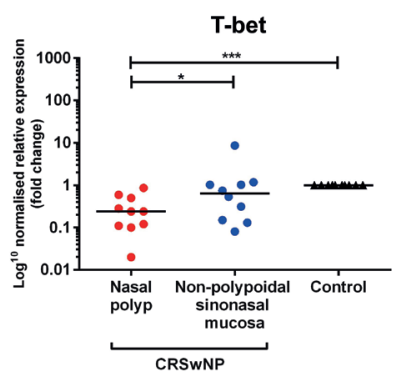

F.

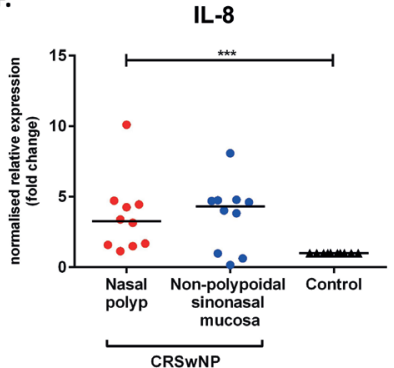

c.

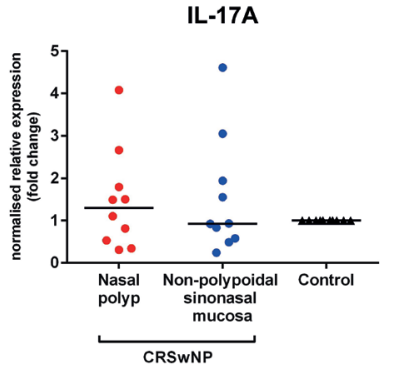

G.

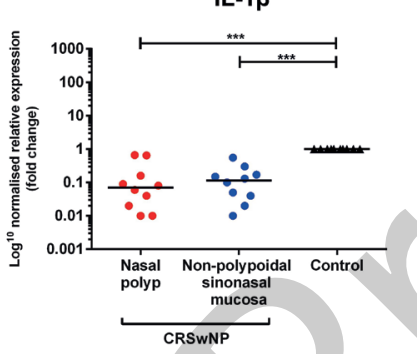

D.

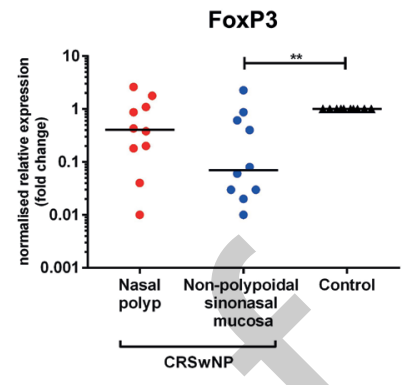

H.

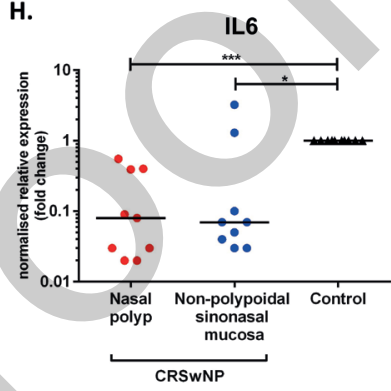

Figure 1. IL-5, T-bet, IL-17A, FoxP3, TLR-4, IL-8, IL-1 $\beta$ and IL-6 normalised relative gene expression ratios within sinonasal mucosal tissue samples. RT-qPCR of IL-5 (A), T-bet (B), IL-17A (C), FoxP3 (D), TLR-4 (E), IL-8 (F), IL-1 $\beta$ (G) and IL-6 (H). Data points represent individual samples and lines represent medians. Significance levels: $p \leq 0.05\left(^{*}\right), p \leq 0.01\left(^{* *}\right)$ and $p \leq 0.001\left(^{* *}\right)$. Experiments involved 10 CRSwNP and 10 Control samples, with RT-qPCR experiments undertaken in duplicate.

\section{Results}

Tissue cytokine gene expression

There was evidence of a Th2 mediated pro-inflammatory response in nasal polyps and non-polypoidal sinonasal mucosa compared to controls (Figure 1A and 1B), with upregulated IL-5 gene expression and downregulated T-bet expression. There was no significant difference in IL-17A gene expression, a Th17 cytokine, across tissue samples (Figure 1C). There was downregulation of FoxP3 expression in nasal polyps and non-polypoidal sinonasal mucosa compared to controls, with the latter reaching statistical significance (Figure 1D).

Toll-like receptor (TLR)-4 was significantly upregulated in nasal polyps and non-polypoidal sinonasal mucosa, in comparison to controls (Figure 1E). IL-8 gene expression was upregulated within non-polypoidal sinonasal mucosa and nasal polyps in comparison to control mucosa, with the latter reaching statistical significance (Figure 1F). IL-1 $\beta$ and IL-6 gene expression were significantly downregulated in both nasal polyps and nonpolypoidal sinonasal mucosa in comparison to controls (Figure $1 \mathrm{G}$ and $1 \mathrm{H})$.

\section{SEB stimulation in a nasal explant model}

There was significant upregulation of IL-5 and IL-17A gene expression, together with IL-5 and IL-17A protein concentration in culture supernatants, following SEB stimulation of nasal polyps (Figures $2 \mathrm{C}$ to F). The protein concentrations of IFNy increased significantly in both control and nasal polyp samples, following
SEB stimulation (Figure 2B). This was not evident when examining the gene expression ratio of T-bet, although there was a significant difference between SEB stimulated controls and SEB stimulated nasal polyps (Figure 2A).

Upon nasal polyp stimulation with SEB, there was significant upregulation of TLR-4 gene expression (Figure $3 \mathrm{~A}$ ) and significant downregulation of IL-1 $\beta$ gene expression (Figure $3 C$ ), with no significant change in IL-8 and IL-6 seen (Figure 3B and D). Control samples showed evidence of upregulated pro-inflammatory cytokines and increased cytokine protein release into the supernatant when nasal polyps were stimulated with SEB (Figures 2 and 3), but these changes only reached statistical significance for IFN $\gamma$ protein concentrations.

\section{Discussion}

Limited studies have compared the immune profiles of nasal polyps with adjacent non-polypoidal sinonasal mucosa from the same patients ${ }^{(25,34-36)}$. Those studies which did, mainly examined the cellular profile of key immune cells in CRSwNP tissue using flow-cytometry, identifying an active Th2 pro-inflammatory response ${ }^{(25,34-36)}$. The present study uses alternative techniques focusing on gene expression levels, providing novel findings that complement previous studies.

Despite their contrasting macroscopic appearances to the naked eye, the non-polypoidal sinonasal mucosa and nasal polyps in CRSwNP patients display strikingly similar immune profiles. Indeed, presented results show that in many cases the non-poly- 

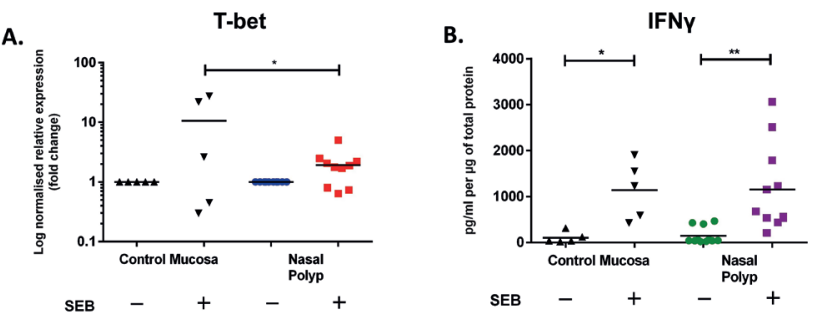

C.

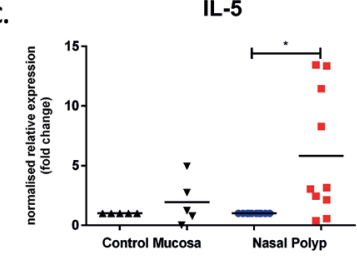

E.
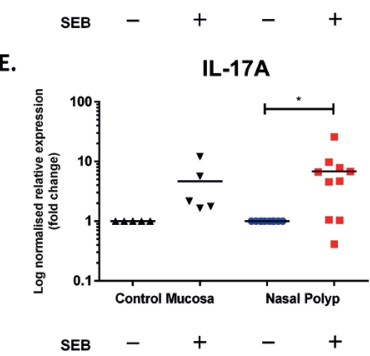

D.

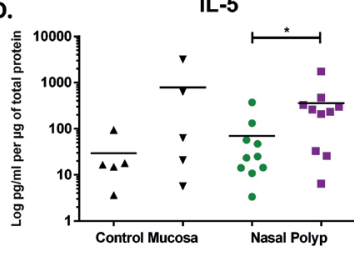

SEB

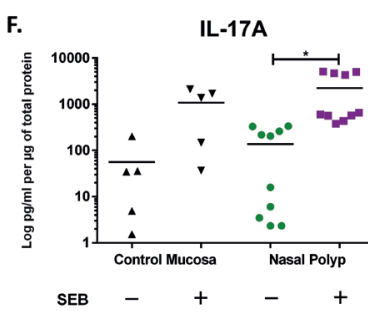

Figure 2. T-cell responses of SEB stimulated sinonasal control mucosa and nasal polyp explants. Normalised relative gene expression (RT-qPCR) of T-bet (A), IL-5 (B) and IL-17A (C) are displayed, together with the corresponding protein concentrations (Luminex) of IFNY (D), IL-5 (E) and IL-17A (F). Data points represent individual samples and lines represent mean values. Significance levels: $p \leq 0.05\left(^{*}\right)$ and $p \leq 0.01\left(^{* *}\right)$. Experiments involved 10 CRSwNP and 5 Control samples, with RT-qPCR experiments undertaken in duplicate and Luminex as single samples. SEB Staphylococcus aureus enterotoxin B.

poidal sinonasal mucosa displays higher pro-inflammatory gene expression levels compared to nasal polyps. This is significant as these are the mucosal areas which may develop over time into frank nasal polyps. Furthermore, these are the same areas which require targeting with topical steroids to reduce transformation into nasal polyps and prevent recurrence following surgical removal.

Whilst reduced levels of $\mathrm{IL}-1 \beta$ have been observed in nasal polyps with high IL-5 expression ${ }^{(37)}$, the significant downregulation of IL-1 $\beta$ and IL- 6 gene expression in CRSwNP samples compared to controls has not been previously reported. IL-1 $\beta$, a cytokine implicated in a number of inflammatory conditions and produced by monocytes and macrophages, is important in the defence against bacterial infections at mucosal surfaces ${ }^{(38)}$. Moreover, when its activity is inhibited in animal models, there is increased susceptibility to bacterial infections including those caused by S. aureus ${ }^{(29,30)}$. This study has revealed IL-1 $\beta$ to be significantly downregulated in CRSwNP samples, which are known to harbour submucosal and intracellular S aureus ${ }^{(18)}$.

A.
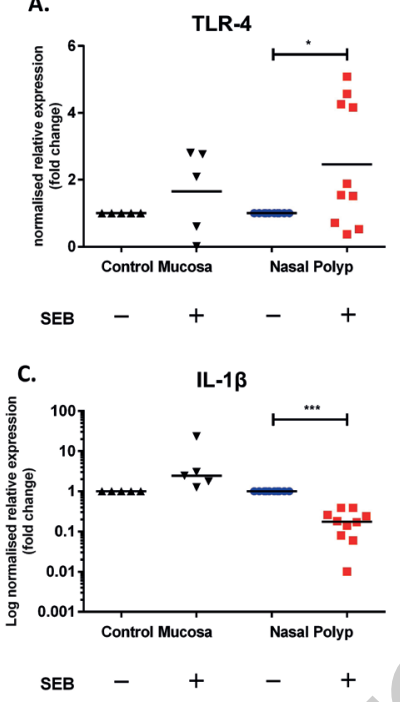

B.

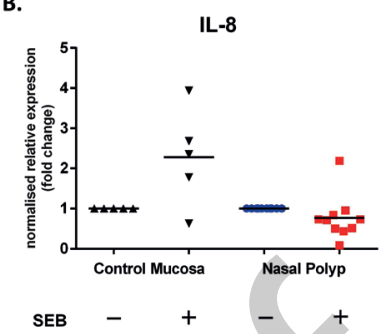

D.

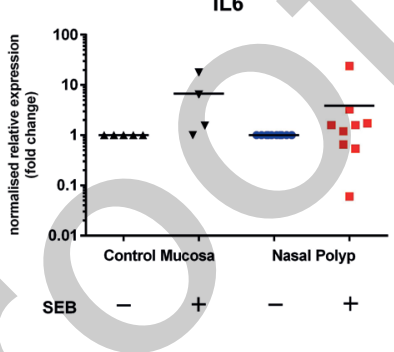

Figure 3. TLR-4, IL-8, IL-1 $\beta$ and IL- 6 gene expression of SEB stimulated sinonasal control mucosa and nasal polyp explants. RT-qPCR of TLR-4 (A), IL-8 (B), IL-1 $\beta$ (C), and IL-6 (D). Data points represent individual samples and lines represent medians. Significance levels: $p \leq 0.05\left(^{*}\right)$ and $p \leq 0.001$ ${ }^{* * *}$. Experiments involved $10 \mathrm{CRSwNP}$ and 5 Control samples, with RT-qPCR experiments undertaken in duplicate. SEB - Staphylococcus aureus enterotoxin B.

Furthermore, IL-1 $\beta$ gene expression was further downregulated when nasal polyps were stimulated with SEB. These results may indicate a disordered anti-bacterial response, on both the tissue and cellular level, which could contribute to enhanced bacterial survival. This is further supported by the findings of IL-6 gene expression, involved in phagocytic cell activation and acute phase response to infection, which was also found to be downregulated in CRSwNP samples. IL-6 deficiency can lead to impaired immunity to viral, parasitic and bacterial infections ${ }^{(31)}$. Combined with upregulation of IL- 8 and TLR-4, these results indicate upregulation of cytokines and toll-like receptors involved in the detection of bacteria and recruitment of defensive cell types, with downregulation of cytokines involved in the immune-mediated clearance of bacteria. This may represent a mechanism by which bacteria subvert the local immune response by recruiting cells (such as mast cells) which they are then able to parasitise, enabling them to evade the immune system thus enhancing their survival.

The potent toxin released by S. aureus, SEB, is known to induce potent pro-inflammatory effects in those with nasal polyps $s^{(3,26,39,}$ 40). Previous studies have shown exacerbation of Th2-mediated inflammation in response to nasal polyps stimulated with $\mathrm{SEB}^{(4)}$. This study has revealed a Th2/Th17-mediated inflammatory response of nasal polyp tissues following SEB stimulation, which has not been reported previously. With a move away from the traditional and rigid classification of CRSWNP as a purely Th2 dri- 
ven process, these results suggest a co-existing Th17 response, evidenced at the gene and protein level. Polyp tissues correlate highly with the intracellular residency of $S$. aureus ${ }^{(18)}$, and this Th17 mediated response which constitutes a defence mechanism against invading pathogens, could provide further support for the role of bacteria in this disease process. It also further confirms the potential inflammatory drive of these tissues, and the propensity for further chronic inflammation.

This study has highlighted the presence of a pro-inflammatory response within the non-polypoidal sinonasal mucosa of CRSwNP patients, together with evidence of both T-regulatory cell dysfunction and disordered bacterial clearance. These may contribute to the enhanced survival of bacteria and the development of a chronic inflammatory state. Furthermore, SEB has been shown to further skew the immune profile of nasal polyps towards the Th2 and Th17 pathways, thus highlighting the ability of bacterial toxins to promote and sustain chronic inflammation. This pro-inflammatory response in seemingly normal looking non-polypoidal sinonasal mucosa reinforces the potential for this mucosa to transform with time into frank polyps, and as such the importance of aggressive and regular anti-inflammatory medical treatment in patients with nasal polyps, including following surgical removal to reduce the risk of polyp growth and recurrence.

\section{Conclusion}

Better understanding of the aetiology of nasal polyps through studies such as this will aid the development of novel therapeutic strategies targeting their formation and growth, thus reducing reliance on antibiotics, corticosteroids and the need for surgical removal. These may include strategies specifically targeting intracellular bacteria, or immunotherapeutic treatments such as anti-IL-5 mAb ${ }^{(41)}$, with the aim of modulating a strongly polarised T-cell response.

\section{Acknowledgements}

We are indebted to all the study participants. We would also to thank Mr Nijaguna Mathad (Consultant Neurosurgeon, University Hospital Southampton NHS Foundation Trust) and Mr Ashok Rokade (Consultant Otorhinolaryngologist and Anterior Skull Base Surgeon, University Hospital Southampton NHS Foundation Trust) for providing the control tissue.

This work was funded by the National Institute for Health Research (NIHR); Rosetrees Trust (A517), Otorhinolaryngological Research Society, Royal Society of Medicine, University of Southampton, British Rhinological Society, and the Royal College of Surgeons of England. This study was supported by the Southampton NIHR Respiratory Biomedical Research Centre, and the Southampton NIHR Wellcome Trust Clinical Research Facility.

\section{Author contriibution}

TCB contributed to the conception and design of the study, the acquisition, analysis and interpretation of all data, and wrote the manuscript. SMH contributed towards the revision of the final manuscript. PGH provided tissue samples, approved and revised the final manuscript. RNA, AFW and SLFP, supervised the study, contributed to the conception and design of the study, were involved in data interpretation, together with revision of the final manuscript. RJS contributed to the conception and design of the study, supervised the study and was involved in writing and critical revision of the manuscript at all stages. All seven authors have read and approved the final version of the manuscript.

\section{Conflict of interest}

None to declare.

\section{References}

1. Fokkens WJ, Lund VJ, Mullol J, et al. European Position Paper on Rhinosinusitis and Nasal Polyps 2012. Rhinol Suppl 2012 $1-298$.

2. Ou J, Wang J, Xu Y, et al. Staphylococcus aureus superantigens are associated with chronic rhinosinusitis with nasal polyps: a meta-analysis. Eur Arch Otorhinolaryngol 2014; 271:2729-36

3. Bachert C, Zhang N, Patou J, van Zele T, Gevaert P. Role of staphylococcal superantigens in upper airway disease. Curr Opin Allergy Clin Immunol 2008; 8: 34-8.

4. Patou J, Gevaert P, Van Zele T, Holtappels $G$, van Cauwenberge $P$, Bachert $C$. Staphylococcus aureus enterotoxin B, protein $\mathrm{A}$, and lipoteichoic acid stimulations in nasal polyps. J Allergy Clin Immunol 2008; 121: 110-5.

5. Derycke L, Eyerich S, Van Crombruggen
$K$, et al. Mixed T helper cell signatures in chronic rhinosinusitis with and without polyps. PLoS One 2014; 9: e97581.

6. Lou H, Meng Y, Piao Y, et al. Cellular phenotyping of chronic rhinosinusitis with nasal polyps. Rhinology 2016; 54: 150-9.

7. Kostamo K, Toskala E, Tervahartiala T, Sorsa T. Role of matrix metalloproteinases in chronic rhinosinusitis. Curr Opin Allergy Clin Immunol 2008; 8: 21-7.

8. Van Bruaene $\mathrm{N}$, Bachert C. Tissue remodeling in chronic rhinosinusitis. Curr Opin Allergy Clin Immunol 2011; 11: 8-11.

9. Watelet JB, Bachert C, Claeys C, Van Cauwenberge P. Matrix metalloproteinases MMP-7, MMP-9 and their tissue inhibitor TIMP-1: expression in chronic sinusitis vs nasal polyposis. Allergy 2004; 59: 54-60.

10. Sachse F, Becker K, von Eiff C, Metze D, Rudack C. Staphylococcus aureus invades the epithelium in nasal polyposis and induces IL-6 in nasal epithelial cells in vitro. Allergy 2010; 65: 1430-7.

11. Li YY, Li CW, Chao SS, et al. Impairment of cilia architecture and ciliogenesis in hyperplastic nasal epithelium from nasal polyps. J Allergy Clin Immunol 2014; 134: 1282-92.

12. Lee JT, Kennedy DW, Palmer JN, Feldman M, Chiu AG. The incidence of concurrent osteitis in patients with chronic rhinosinusitis: a clinicopathological study. Am J Rhinol 2006; 20: 278-82.

13. Telmesani LM, Al-Shawarby M. Osteitis in chronic rhinosinusitis with nasal polyps: a comparative study between primary and recurrent cases. Eur Arch Otorhinolaryngol 2010; 267: 721-4.

14. Cho GS, Moon BJ, Lee BJ, et al. High rates of detection of respiratory viruses in the nasal washes and mucosae of patients with chronic rhinosinusitis. J Clin Microbiol 2013; 51: 979-84. 
15. Tan NCW, Foreman A, Jardeleza C, Douglas $\mathrm{R}$, Tran H, Wormald PJ. The multiplicity of Staphylococcus aureus in chronic rhinosinusitis: Correlating surface biofilm and intracellular residence. Laryngoscope 2012 122: 1655-60.

16. Tan NCW, Foreman A, Jardeleza C, Douglas R, Vreugde S, Wormald P-J. Intracellular Staphylococcus aureus: the Trojan horse of recalcitrant chronic rhinosinusitis? Int Forum Allergy Rhinol 2013; 3: 261-6.

17. Foreman A, Holtappels G, Psaltis AJ et al. Adaptive immune responses in Staphylococcus aureus biofilm-associated chronic rhinosinusitis. Allergy 2011; 66 1449-56.

18. Hayes SM, Howlin R, Johnston DA, et al Intracellular residency of Staphylococcus aureus within mast cells in nasal polyps: A novel observation. J Allergy Clin Immunol 2015; 135: 1648-51.

19. Sabirov A, Hamilton RG, Jacobs JB, Hillman DE, Lebowitz RA, Watts JD. Role of local immunoglobulin E specific for Alternaria alternata in the pathogenesis of nasal polyposis. Laryngoscope 2008; 118: 4-9.

20. Sproson EL, Thomas KM, Lau LC, Harries PG, Howarth PH, Salib RJ. Common airborne fungi induce species-specific effects on upper airway inflammatory and remodelling responses. Rhinology 2016; 54: 51-5.

21. Tomassen P, Vandeplas G, Van Zele T, et al Inflammatory endotypes of chronic rhinosinusitis based on cluster analysis of biomarkers. J Allergy Clin Immunol 2016; 137: 144956.e4.

22. Bachert C, Akdis CA. Phenotypes and Emerging Endotypes of Chronic Rhinosinusitis. J Allergy Clin Immunol Pract 2016; 4: 621-8.

23. Lou H, Meng Y, Piao Y, et al. Cellular phenotyping of chronic rhinosinusitis with nasal polyps. Rhinology 2016; 54: 150-9.

24. Wang X, Zhang N, Bo Ms M, et al. Diversity of $\mathrm{TH}$ cytokine profiles in patients with chronic rhinosinusitis: A multicenter study in Europe, Asia, and Oceania. J Allergy Clin
Immunol 2016; 138: 1344-1353.

25. Miljkovic D, Psaltis A, Wormald PJ, Vreugde S. T regulatory and Th17 cells in chronic rhinosinusitis with polyps. Int Forum Allergy Rhinol 2016; 6: 826-34.

26. Bachert C, Zhang N, van Zele T, Gevaert P, Patou J, van Cauwenberge $P$. Staphylococcus aureus enterotoxins as immune stimulants in chronic rhinosinusitis. Clinical allergy and immunology 2007; 20: 163-75.

27. Kolaczkowska E, Kubes P. Neutrophil recruitment and function in health and inflammation. Nat Rev Immunol 2013: 13: 159-75.

28. Nilsson G, Mikovits JA, Metcalfe DD, Taub DD. Mast cell migratory response to interleukin-8 is mediated through interaction with chemokine receptor CXCR2/ Interleukin-8RB. Blood 1999; 93: 2791-7.

29. LaRock CN, Todd J, LaRock DL, et al. IL-1 beta is an innate immune sensor of microbial proteolysis. Sci Immunol 2016; 1

30. Ali $A, N a$ M, Svensson $M N$, et al. $\mid L^{-1}$ Receptor Antagonist Treatment Aggravates Staphylococcal Septic Arthritis and Sepsis in Mice. PLoS One 2015; 10: e0131645.

31. Hunter CA, Jones SA. IL-6 as a keystone cytokine in health and disease. Nat Immunol 2015; 16: 448-57.

32. Lund VJ, Mackay IS. Staging in rhinosinusitus. Rhinology 1993; 31: 183-4

33. Schmittgen TD, Livak KJ. Analyzing real-time PCR data by the comparative C(T) method. Nat Protoc 2008; 3: 1101-8.

34. Ho J, Bailey M, Zaunders J, et al. Cellular comparison of sinus mucosa vs polyp tissue from a single sinus cavity in chronic rhinosinusitis. Int Forum Allergy Rhinol 2015; 5: 14-27.

35. Morinaka S, Nakamura H. Inflammatory cells in nasal mucosa and nasal polyps. Auris Nasus Larynx 2000; 27: 59-64.

36. Hao J, Pang YT, Wang DY. Diffuse mucosal inflammation in nasal polyps and adjacent middle turbinate. Otolaryngol Head Neck Surgery 2006; 134: 267-75.

37. Krysko O, Holtappels G, Zhang N, et al.
Alternatively activated macrophages and impaired phagocytosis of $\mathrm{S}$. aureus in chronic rhinosinusitis. Allergy 2011; 66: 396403.

38. Netea MG, Simon A, van de Veerdonk $F$ Kullberg BJ, Van der Meer JW, Joosten LA. IL-1 beta processing in host defense: beyond the inflammasomes. PLoS Pathog 2010; 6:e1000661

39. Van Zele T, Gevaert P, Watelet JB, et al. Staphylococcus aureus colonization and $\lg \mathrm{E}$ antibody formation to enterotoxins is increased in nasal polyposis. J Allergy Clin Immunol 2004; 114: 981-3.

40. Zhang N, Gevaert P, van Zele T, et al. An update on the impact of Staphylococcus aureus enterotoxins in chronic sinusitis with nasal polyposis. Rhinology 2005; 43: 162-8.

41. Gevaert P, Van Bruaene N, Cattaert T, et al. Mepolizumab, a humanized anti-IL-5 mAb, as a treatment option for severe nasal polyposis. J Allergy Clin Immunol 2011; 128 : 989-95.e1-8.

Rami J. Salib

Associate Professor of Rhinology \& Consultant Otorhinolaryngologist School of Clinical \& Experimental Sciences

Department of Otorhinolaryngology

/ Head \& Neck Surgery,

University Hospital Southampton

NHS Foundation Trust

Tremona Road

Southampton, SO16 6YD

United Kingdom

Telephone: +44 238054 0276;

Fax: +44 2380825688 ;

E-mail: R.J.Salib@soton.ac.uk

RT-qPCR primer sequences.

\begin{tabular}{llll} 
Gene name & Manufacturer & Sense primer & Anti-sense primer \\
\hline T-bet & Primerdesign & CAGCACCAGACAGAGATGATC & GCCACAGTAAATGACAGGAATG \\
IL-5 & Primerdesign & CCCAGAGATAAGGTTCGTTCT & CAACCCCAGCATCTAGCATAG \\
IL-17A & Primerdesign & CCTCAGATTACTACAACCGATCC & CACTTTGCCTCCCAGATCAC \\
\hline FoxP3 & Primerdesign & CAGCACATTCCCAGAGTTCCT & GCGTGTGAACCAGTGGTAGAT \\
\hline TLR-4 & Primerdesign & TGCCTTCACTACAGAGACTTTATTCC & GACACCACAACAATCACCTTTCG \\
IL-8 & Primerdesign & CAGAGACAGCAGAGCACAC & AGCTTGGAAGTCATGTTTACAC \\
IL-6 & Primerdesign & GCAGAAAACAACCTGAACCTT & ACCTCAAACTCCAAAAGACCA \\
IL-1 $\beta$ & Primerdesign & TGGCAATGAGGATGACTTGTTC & CTGTAGTGGTGGTCGGAGATT
\end{tabular}

RT-qPCR Housekeeping gene amplicon context information: Human ACTB, Accession number: NM_001101, Anchor Nucleotide position: 1195,

Context sequence length: $106 \mathrm{bp}$ 